\title{
Generation Method of Digital Disruptive Pattern
}

\author{
Jun $\mathrm{Yu}^{1, \mathrm{a}}$, Zhiyi Hu${ }^{2, \mathrm{~b}}$ \\ ${ }^{1}$ Xi'an Technological University, Xi'an, China \\ ${ }^{2}$ Architectural Engineering Research Institute, Xi'an, China \\ ajyu0117@163.com, bhu016v7@yahoo.cn
}

Keywords: digital disruptive pattern, background image, main color, camouflage effect

\begin{abstract}
This paper presents a new method which can generate a digital camouflage pattern based on the background image of a target. It quantizes the background color, and extracts the main color, followed that determines the size of a mosaic block. By filling the blocks with the main color, the digital disruptive pattern is generated. In order to test the camouflage effect, the designed pattern is printed on the tent cloth, and the tent is placed in the actual background. The experimental result shows that, the digital camouflage is easier to blend with the natural background than the traditional camouflage, and achieves a good effect of the optical camouflage.
\end{abstract}

\section{Introduction}

With the development of high-tech detection means including microwaves, lasers and satellites in the modern information warfare, the abilities of obtaining the battlefield information is growing, as well as the requirements for military camouflage technology becomes higher and higher.

The traditional camouflage has been developed and applied for nearly one century since the World War II. It has played a significant role in protecting military targets. The traditional camouflage is mainly composed of irregular spots or stripes, whose boundaries between different colors are smooth and clear. These characteristics conduces to strong visual discrimination, and therefore be fit for close range, low-resolution optical reconnaissance and surveillance. Digital camouflage is a pattern devised by using small micro patterns, as opposed to larger patterns for effective disruption. Digital patterns are designed mostly with mathematical algorithms, which simulate the appearance of digital images taken from a specific environment.

This paper presents a new method of digital camouflage generation. It extracts the features from the background image of a military objective, followed that calculate the mosaic size. Finally the digital disruptive pattern is generated. The generated pattern overcomes the shortcomings of smooth boundaries, and makes the edges blur between different colors. Therefore, the digital disruptive pattern has a good mixing effect, and more easily integrated with the target background.

\section{Design Process of Digital Disruptive Pattern}

The design of digital camouflage should be based on the background information. It includes two steps those are extracting main colors from the background and designing camouflage spots. The Fig. 1 shows the detailed flow chart of digital camouflage design.

The first step is to read the background data of the objective image. After that, there are two hands of things to do. On the one hand, the background image is conversed from RGB color space to HIS color space, follows that measures and reduces the number of colors, finally extracts main background colors; On the other hand, the mosaic block size is calculated, and then the layout spots is designed. Then the spots are filled by different colors, and consequently a digital disruptive pattern is generated. As a result, the method is easy to implement, as well as can accurately extract the main colors and build a digital pattern. 


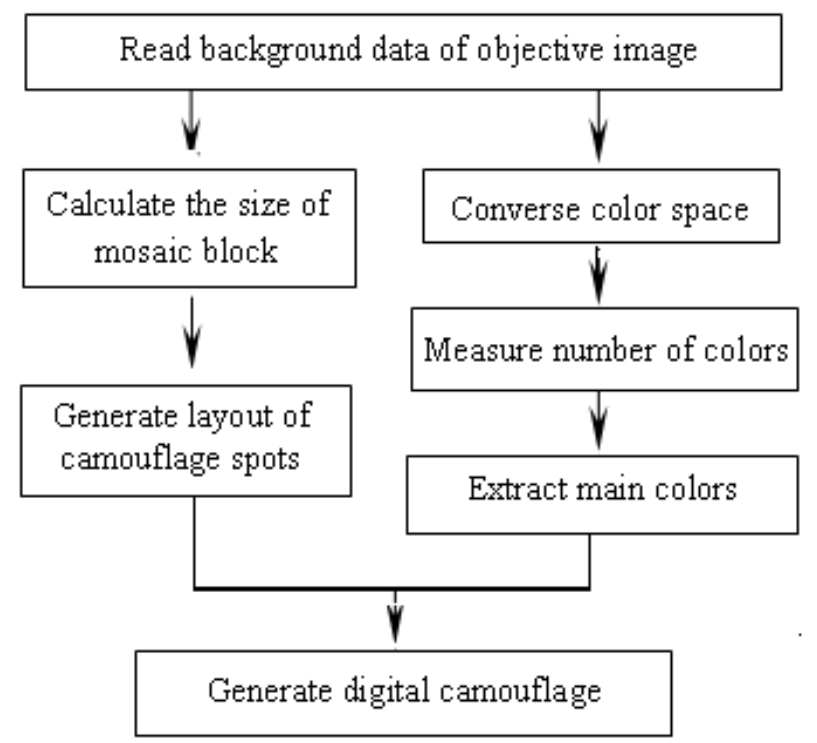

Fig.1 Flow chart of digital camouflage design

\section{Generation Algorithm of Digital Disruptive Pattern}

Color Spaces Conversion. At present, there are two kinds of common color spaces. One is the RGB color space, which is faced to hardware devices and easy to display. The other is the HSI color space for human vision. The HSI space is usually selected in the camouflage design [1].

The RGB color space can easily converse to HSI color space. Firstly, we integrate R, G, B component to the rang $[0,1]$. Then we calculate the corresponding $\mathrm{H}, \mathrm{S}, \mathrm{I}$ components according to the formula (1) (3).

$$
\begin{array}{r}
I=\frac{1}{3}(R+G+B) \\
\quad S=1-\frac{3}{(R+G+B)}[\min (R, G, B)] \\
H=\arccos \left\{\frac{[(R-G)+(R-B)] / 2}{\left[(R-B)^{2}+(R-B)(G-B)^{1 / 2}\right]}\right\}
\end{array}
$$

If the value of $\mathrm{S}, \mathrm{I}$ is in $[0,1]$, then $\mathrm{R}, \mathrm{G}$ and $\mathrm{B}$ are also in $[0,1]$. The formula for conversing HSI space to RGB space is as follows.

If $\mathrm{H}$ is in $[0,120]$, then

$$
\begin{gathered}
R=I\left[1+\frac{S \cos H}{\cos \left(60^{\circ}-H\right)}\right] \\
B=I(1-S) \\
G=3 I-(B+R)
\end{gathered}
$$

If $\mathrm{H}$ is in $[120,240]$, then

$$
\begin{gathered}
R=I(1-S) \\
G=I\left[1+\frac{S \cos \left(H-120^{\circ}\right)}{\cos \left(180^{\circ}-H\right)}\right]
\end{gathered}
$$




$$
B=3 I-(G+R)
$$

If $H$ is in $[240,360]$, then

$$
\begin{aligned}
& G=I(1-S) \\
& B=I\left[1+\frac{S \cos \left(H-240^{\circ}\right)}{\cos \left(300^{\circ}-H\right)}\right] \\
& R=3 I-(G+B)
\end{aligned}
$$

Calculating Color Histogram and Extracting Main Colors. The gray image can directly extract main colors from its gray-level histogram [2,3]. There are three components within a color image. The number of each component is very great. For example, a 24-bit color image can express $16,777,216$ colors. The human eyes can distinguish ten million kinds of colors. Therefore, we have to reduce the number of colors so as to decrease the computing workload. In order to reduce the computation workload, the number of colors should be decreased to 300 or so. The values of S and I are reverted to the area $[0,255]$. According to the experimental result, the HSI space is quantified.

if $\mathrm{I} \in[\mathrm{m} \times 51, \quad(\mathrm{~m}+1) \times 51]$, then $\mathrm{I}=\mathrm{m} \quad(\mathrm{m}=0,1,2,3,4)$.

if $S \in[n \times 51, \quad(n+1) \times 51]$, then $S=n \quad(n=0,1,2,3,4)$.

if $\mathrm{H} \in[\mathrm{k} \times 22, \quad(\mathrm{k}+1) \times 22]$, then $\mathrm{H}=\mathrm{k} \quad(\mathrm{k}=0,1,2, \ldots, 11)$.

The above three components are unified and quantified as follows.

$\mathrm{W}=25 \mathrm{H}+5 \mathrm{~S}+\mathrm{I}$

In the formula (13), the range of $\mathrm{W}$ is in $[0,299]$, which is respectively corresponded to the HSI components. That is to say, a group of HSI components is corresponded to an only W value. The different $\mathrm{W}$ value is also corresponded with an only group of HSI components.

The algorithm of extracting main colors is as follows.

- Sets one-dimensional array $W[300]$, and defines initial value $W[n]=0$. Here $n=(0,1, \ldots, 299)$.

- Calculates the value of each HIS component based on the RGB value for the original image.

- Calculates M, N and K, those are corresponding to the HIS components.

- Calculates the value of $\mathrm{W}$ from $\mathrm{M}, \mathrm{N}$ and $\mathrm{K}$. It satisfies the formula $\mathrm{W}[\mathrm{n}]=\mathrm{W}[\mathrm{n}]+1$.

- Extracts the largest five values as the main colors from the one-dimensional array W[300].

Calculating Size of Mosaic Block. According to the principle of human vision [4], the distinction capacity of human eyes is about 1 cent (that is about 0.017 degree). If a point is less than 1 cent in the apparent angle of eyes, then an observer can not find this point. If an included angle, formed by a line based on two adjacent points and an eye, is less than 1 cent, it is impossible to distinguish these two points for human eyes. Assumes that an observer makes a reconnaissance in D meters, the formula for calculating the size of mosaic block is as follows.

$\mathrm{d}=\tan 0.017 * \mathrm{D}$

Here, $\mathrm{d}$ is the size of mosaic block. $\mathrm{D}$ is the observing distance. The value of $\tan 0.017$ is about 0.00029 . Thus the formula can be simplified as $d=0.00029 * \mathrm{D}$.

Generating Digital Camouflage. In the process of generating the digital camouflage, two factors must be considered [5]. Firstly, the pixels should be discrete. From visible lights to infrared wavebands, a group of unorderly pixels are observed using the detection equipment. An observer can neither identify the edge of pixel clusters, nor distinguish per pixel from the surrounding pixels. Thus the target can not be recognized. Secondly, the number of filled main colors is assured. During the process of filling main colors, if a kind of color is filled too much, the camouflage effect is influenced. Thus the colors are necessary to be calculated. 
Assumes that there are five kinds of main colors, those are respectively c1, c2, c3, c4 and c5. The corresponding number of colors are $\mathrm{a}, \mathrm{b}, \mathrm{c}, \mathrm{d}$ and e. During the filling process, the occurrence probabilities of these colors in the pattern are respectively as follows.

$$
\mathrm{p}(\mathrm{c} 1)=\mathrm{a} / \mathrm{m}, \mathrm{p}(\mathrm{c} 2)=\mathrm{b} / \mathrm{m}, \mathrm{p}(\mathrm{c} 3)=\mathrm{c} / \mathrm{m}, \mathrm{p}(\mathrm{c} 4)=\mathrm{d} / \mathrm{m}, \mathrm{p}(\mathrm{c} 5)=\mathrm{e} / \mathrm{m}
$$

Here, $\mathrm{m}=\mathrm{a}+\mathrm{b}+\mathrm{c}+\mathrm{d}+\mathrm{e}$.

According to the above description, the generation algorithm is as follows.

The first step is to determine the length $d$ of a mosaic block. Assumes that the number of the square-blocks is $\mathrm{N}=W \times H$. Here $W$ is the number of blocks per row. $H$ is the number of blocks per column. $\mathrm{N}$ is the total number of the blocks.

The second step is to determine main colors, those are c1, c2, c3, c4 and c5. Followed that to calculate their occurrence probability, those are $\mathrm{p}(\mathrm{c} 1), \mathrm{p}(\mathrm{c} 2), \mathrm{p}(\mathrm{c} 3), \mathrm{p}(\mathrm{c} 4)$ and $\mathrm{p}(\mathrm{c} 5)$.

The third step is to paint the block with the color $\mathrm{c} 1$. The block contains $\mathrm{N} \times \mathrm{p}(\mathrm{c} 1)$ pixels.

The fourth step is to fill the other blocks respectively with $\mathrm{c} 2, \mathrm{c} 3, \mathrm{c} 4$ and $\mathrm{c} 5$ in the same way.

Eventually, we get the digital camouflage pattern.

\section{Experimental results and analysis}

As described previously, the experiment uses the algorithm to generate a digital camouflage pattern. In the Fig.2, picture (a) shows a traditional camouflage pattern. Picture (b) is a digital camouflage pattern generated by this algorithm. These two patterns are similar on color types, color proportion and spot size. The main difference between (a) and (b) is the shape and distribution. For example, the two patterns are placed in the same background, and then the regular outlines are extracted. Picture (c) is extracted from (a), and (d) is from (b). The small white boxes are regular outline. As a result, the number of regular outlines in (c) is much more than in (d). It means that, the probability of targetfound are greatly reduced in (d) than in (c).

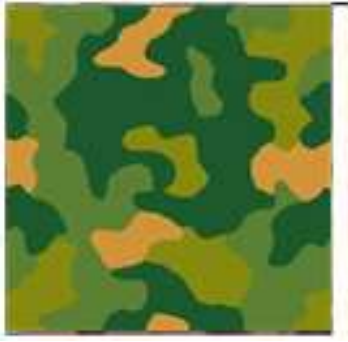

(a)

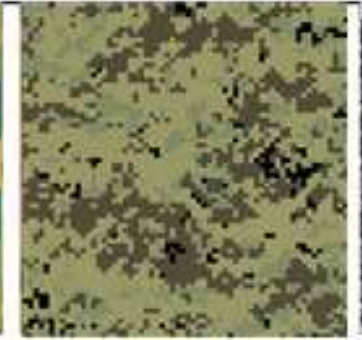

(b)

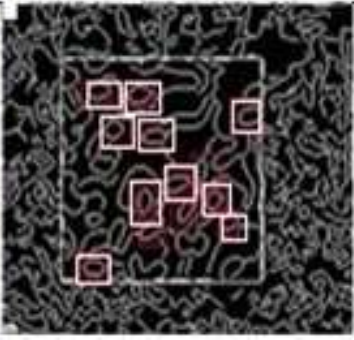

(c)

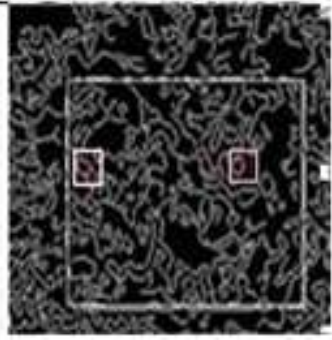

(d)

Fig. 2 Comparison between traditional camouflage and digital camouflage. (a) traditional camouflage; (b) digital camouflage; (c) regular pictures extracted from (a); (d) regular pictures extracted from (b)

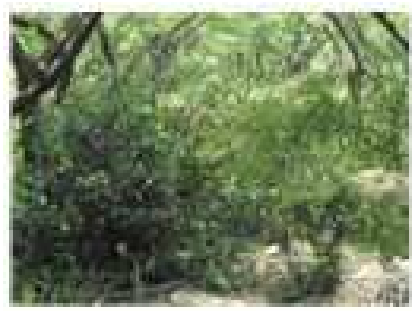

(a)

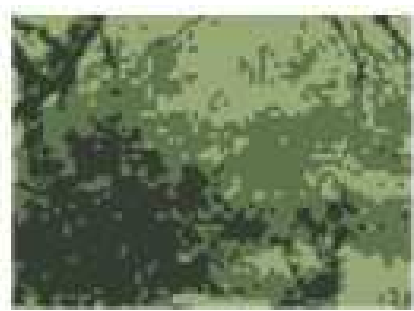

(b)

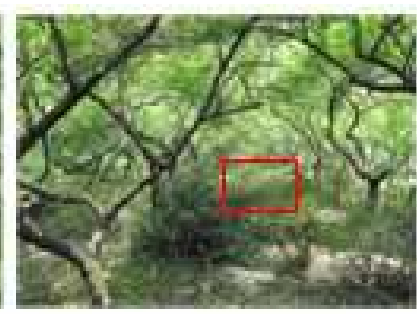

(c)

Fig.3 Woodland camouflage.

(a) woodland background; (b) designed digital camouflage; (c) camouflaged effect 
Another experiment is showed in Fig.3. Picture (a) is an original background in woodland. Picture (b) is a digital camouflage pattern generated by our method. Picture (c) shows the camouflage effect when the designed camouflage pattern is applied on the original background. The red pane makes a sign of the target. Apparently, the digital camouflage is better integrated with the background than the traditional camouflage.

\section{Conclusion}

This paper presents a new method of generating a digital camouflage based on the natural background. It firstly quantifies the HSI color space, then determines the size of a mosaic block, followed that fills the main colors into the spots, finally generates a digital camouflage pattern. As shown in the comparison experiment, the regular outlines extracted from a digital camouflage decreases by $70 \% \sim 80 \%$ compared with a traditional camouflage. As shown in the experiment of woodland camouflage, the generated digital camouflage is easier to blend with the natural background than the traditional camouflage. Therefore, it can achieve a good effect of the optical camouflage.

\section{Acknowledgement}

The financial support of General Logistics Department (Project No. AY111C001) and Education Department of Shaanxi Provincial Government (Project No.11JK1043) are gratefully acknowledged.

\section{References}

[1] T. Acharya. Image Processing:Principles and Applications. Beijing: Tsinghua University Press, 2007, pp.104-158.

[2] R.C.Gonzalez, R.E.Woods. Digital Image Processing (2nd Edition). Addison Wesley Publishing Company, 2002, pp.68-124.

[3] Y.Xu. Camouflage color selection based on dominant color extraction. Opto-Electronic Engineering, 2007, vol.34, pp.100-103.

[4] G.S. Linda, G.C. Stockman. Computer Vision. Prentice Hall, 2001, pp.31-57.

[5] Y.P. Yao. Camouflage of Army equipment. Military Science Press, 2006, pp.125-160. 